\title{
Do variations in insulin sensitivity and insulin secretion in pregnancy predict differences in obstetric and neonatal outcomes?
}

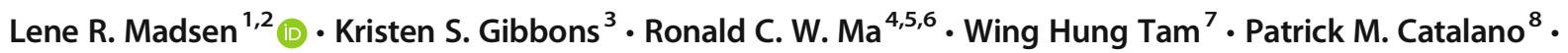 \\ David A. Sacks ${ }^{9} \cdot$ Julia Lowe $^{10} \cdot$ H. David McIntyre ${ }^{2,3}$ (D)
}

Received: 28 May 2020 / Accepted: 15 September 2020 / Published online: 6 November 2020

(C) Springer-Verlag GmbH Germany, part of Springer Nature 2020

\begin{abstract}
Aims/hypothesis Gestational diabetes mellitus (GDM) is generally defined based on glycaemia during an OGTT, but aetiologically includes women with defects in insulin secretion, insulin sensitivity or a combination of both. In this observational study, we aimed to determine if underlying pathophysiological defects evaluated as continuous variables predict the risk of important obstetric and neonatal outcomes better than the previously used dichotomised or categorical approaches.

Methods Using data from blinded OGTTs at mean gestational week 28 from five Hyperglycemia and Adverse Pregnancy Outcome study centres, we estimated insulin secretion (Stumvoll first phase) and sensitivity (Matsuda index) and their product (oral disposition index [DI]) in 6337 untreated women (1090 [17.2\%] with GDM as defined by the International Association of Diabetes and Pregnancy Study Groups). Rather than dichotomising these variables (i.e. GDM yes/no) or subtyping by insulin impairment, we related insulin secretion and sensitivity as continuous variables, along with other maternal characteristics, to obstetric and neonatal outcomes using multiple regression and receiver operating characteristic curve analysis.

Results Stratifying by GDM subtype offered superior prediction to GDM yes/no only for neonatal hyperinsulinaemia and pregnancy-related hypertension. Including the DI and the Matsuda score significantly increased the area under the receiver operating characteristic curve (AUROC) and improved prediction for multiple outcomes (large for gestational age [AUROC 0.632], neonatal adiposity [AUROC 0.630], pregnancy-related hypertension [AUROC 0.669] and neonatal hyperinsulinaemia [AUROC 0.688]). Neonatal hypoglycaemia was poorly predicted by all models. Combining the DI and the Matsuda score with maternal characteristics substantially improved the predictive power of the model for large for gestational age, neonatal adiposity and pregnancy-related hypertension.

Conclusion/interpretation Continuous measurement of insulin secretion and insulin sensitivity combined with basic clinical variables appeared to be superior to GDM (yes/no) or subtyping by insulin secretion and/or sensitivity impairment in predicting obstetric and neonatal outcomes in a multi-ethnic cohort.
\end{abstract}

Supplementary Information The online version contains supplementary material available at https://doi.org/10.1007/s00125-02005323-0.

Lene R. Madsen

leemas@rm.dk

1 Steno Diabetes Center Aarhus, Aarhus University Hospital, Aarhus, Denmark

2 Danish Diabetes Academy, Odense University Hospital, Odense, Denmark

3 Department of Medicine, Regional Mater Research Institute, The University of Queensland, South Brisbane, QLD, Australia

4 Department of Medicine and Therapeutics, The Chinese University of Hong Kong, Hong Kong SAR, China

5 Li Ka Shing Institute of Health Sciences, The Chinese University of Hong Kong, Hong Kong SAR, China
6 Chinese University of Hong Kong-Shanghai Jiao Tong University Joint Research Centre in Diabetes Genomics and Precision Medicine, Hong Kong Institute of Diabetes and Obesity, The Chinese University of Hong Kong, Hong Kong SAR, China

7 Department of Obstetrics and Gynecology, The Chinese University of Hong Kong, Hong Kong SAR, China

8 Department of Obstetrics and Gynecology, Mother Infant Research Institute, Tufts Medical Center, Boston, MA, USA

9 Department of Research and Evaluation, Kaiser Permanente Southern California, Pasadena, CA, USA

10 University of Newcastle, Newcastle, NSW, Australia 


\section{Research in context}

\section{What is already known about this subject?}

- Gestational diabetes mellitus (GDM) carries risks in terms of both obstetric and neonatal outcomes, and a lifetime risk of type 2 diabetes for the mother

- The term 'GDM' covers women with impairments in insulin secretion and insulin sensitivity, but the impairments are closely related

- In previous studies, women with GDM due to impaired insulin sensitivity had the highest risk of poor obstetric and neonatal outcomes

\section{What is the key question?}

- Do measures of insulin secretion and insulin sensitivity, measured continuously rather than being divided into arbitrary categories, relate independently to obstetric and neonatal outcomes, both before and after correction for demographic and clinical characteristics?

\section{What are the new findings?}

- GDM subtyping by insulin impairment offered superior prediction to GDM 'yes/no' only for neonatal hyperinsulinaemia and pregnancy-related hypertension. Adding the continuous oral disposition index and the Matsuda score significantly increased the area under the receiver operating characteristic curve and improved prediction for most outcomes

- Combining the oral disposition index and Matsuda score with maternal characteristics greatly enhanced the predictive power for offspring being large for gestational age, neonatal adiposity and pregnancy-related hypertension

How might this impact on clinical practice in the foreseeable future?

- Using continuous measurements in combination with clinical characteristics could aid in predicting the risk of obstetric and neonatal outcomes on a personalised level

Keywords Caesarean delivery $\cdot$ Gestational diabetes $\cdot$ HAPO study $\cdot$ Insulin secretion · Insulin sensitivity $\cdot$ Large for gestational age $\cdot$ Neonatal outcome $\cdot$ Obstetric outcome $\cdot$ Oral disposition index $\cdot$ Preterm delivery

\author{
Abbreviations \\ AUROC Area under the receiver operating characteristic \\ curve \\ DI Oral disposition index \\ GDM Gestational diabetes mellitus \\ GWG Gestational weight gain \\ HAPO Hyperglycemia and Adverse Pregnancy Outcome \\ IADPSG International Association of Diabetes and \\ Pregnancy Study Groups \\ LGA Large for gestational age \\ ROC Receiver operating characteristic
}

\section{Introduction}

The prevalence of gestational diabetes mellitus (GDM), defined as diabetes diagnosed during the second or third trimester of pregnancy that is not clearly overt diabetes prior to gestation [1], is increasing [2]. GDM prevalence follows the prevalence of obesity and type 2 diabetes in a given country, and the obesity epidemic amongst fertile women in part explains the increasing prevalence of GDM [3]. Furthermore, the International Association of Diabetes and Pregnancy Study Groups (IADPSG) criteria for GDM, which require only one abnormal glucose value on the oral glucose tolerance test (OGTT) for diagnosis, have also contributed to the higher prevalence [4]. GDM carries risks for the mother in terms of both immediate pregnancy complications, especially pregnancy-related hypertension and the need for a Caesarean birth, and her lifetime risk of type 2 diabetes and cardiovascular disease [5]. For the offspring, there are increased shortterm risks of being large for gestational age (LGA) with increased adiposity, preterm delivery, shoulder dystocia and neonatal hypoglycaemia, as well as long-term risks of obesity and abnormal glucose metabolism [3].

In routine clinical practice, GDM is generally defined solely by whether one or more glucose values during an OGTT equal or exceed variable but predefined thresholds. It has previously been suggested that distinct aetiological subtypes exist within the broad 'diagnosis' of GDM, with a proposed 
division into women with predominant defects in insulin sensitivity, those with defects in insulin secretion and those with a combination of both [6]. Furthermore, it has been suggested that not all subtypes carry the same risk of pregnancy complications for the mother and offspring. In a study by Powe et al, women with insulin sensitivity defects had larger offspring and a higher rate of Caesarean delivery (persisting after adjustment for maternal BMI and other confounders) than those with normal glucose tolerance; while the pregnancies of women with GDM caused by insulin secretion defects did not differ from those of women without GDM with respect to these outcomes [6]. Benhalima et al, using a different classification framework from Powe et al, reported that women with GDM with predominant impaired insulin sensitivity had a higher risk of Caesarean delivery, neonatal hypoglycaemia and preterm delivery, but no increase in the risk of an LGA neonate [7]. The authors of both of these papers proposed arbitrary criteria for GDM subtypes using estimates of insulin sensitivity and secretion. These previous studies had important limitations, as noted by the authors. First, both included predominantly white women, so generalisability to other ethnic groups is uncertain. Second, participant numbers in both studies were small. Finally, all women with GDM received treatment (12-42\% requiring insulin), potentially confounding associations between baseline clinical subtypes and outcomes [8].

The Hyperglycemia and Adverse Pregnancy Outcome (HAPO) study [9] clearly demonstrated continuous relationships between OGTT fasting, $1 \mathrm{~h}$ and $2 \mathrm{~h}$ glucose values and a wide range of pregnancy outcomes. We therefore hypothesised that measures of insulin secretion and insulin sensitivity, measured continuously rather than being divided into arbitrary categories, would be independently related to pregnancy outcomes, both before and after correction for demographic and clinical characteristics.

\section{Methods}

The original HAPO study protocol was approved by the institutional review boards of each centre and has been published in detail [10]. HAPO was a blinded epidemiological study. No glucose measurements were revealed to caregivers or participants and no participants received treatment for GDM during pregnancy. All participants gave written informed consent. For the current study, we included data from $75 \mathrm{~g}$ OGTTs performed between 24 and 32 weeks of gestation on women of varying ethnicities from five HAPO study centres (Bellflower, CA, USA; Cleveland, OH, USA; Brisbane, QLD, Australia; Newcastle, NSW, Australia; Hong Kong, China) [4]. We excluded participants $(n=60)$ who were unblinded in the original cohort [10] or who had missing data on key variables. We analysed demographic and clinical characteristics, including (recorded at the OGTT visit): mean OGTT $z$ score (fasting, $1 \mathrm{~h}$ and $2 \mathrm{~h}$ results standardised and then the mean calculated); maternal age, height and parity ( 0 or $\geq 1$ ); BMI at OGTT, grouped as underweight (BMI $<22.6 \mathrm{~kg} / \mathrm{m}^{2}$ ), normal weight ( 22.6 to $<28.5 \mathrm{~kg} / \mathrm{m}^{2}$ ), overweight $\left(28.5\right.$ to $\left.<33.0 \mathrm{~kg} / \mathrm{m}^{2}\right)$ and obese $\left(\geq 33.0 \mathrm{~kg} / \mathrm{m}^{2}\right)$ using categories defined for the OGTT visit in the entire HAPO cohort [11, 12]; smoking; alcohol consumption; hypertensive disorders in pregnancy (systolic BP $>140 \mathrm{mmHg}$ or diastolic BP $>90 \mathrm{mmHg}$ ); family history of diabetes and family history of hypertension (first-degree relative); self-reported ethnicity (white, black, Hispanic, Asian or other); and HAPO study centre. We defined gestational weight gain $(G W G)$ as the difference between self-reported pre-pregnancy weight and weight at last visit; GWG was then categorised according to the Institute of Medicine guidelines using a combination of pre-pregnancy BMI and GWG, as previously reported for a North American subcohort of the HAPO study [13]. At time of birth, the following outcome variables were considered: LGA (defined as $>90$ th centile as per the primary HAPO analyses [9]; a newborn in the HAPO study was considered to have a birthweight $>90$ th percentile if the actual birthweight was greater than the estimated 90th percentile for the baby's sex, gestational age, ethnicity, HAPO centre and maternal parity), neonatal hyperinsulinaemia (cord C-peptide $>90$ th centile $[1.7 \mu \mathrm{g} / \mathrm{l}]$ as per the primary HAPO analyses [9]), neonatal hypoglycaemia (neonatal $2 \mathrm{~h}$ glucose $<10$ th centile of the HAPO cohort [2.2 mmol/l]), neonatal adiposity (per cent body fat $>90$ th centile) and sum of skinfolds $>90$ th centile, primary Caesarean delivery and preterm birth ( $<37$ weeks' gestation). Independent variables had less than $5 \%$ missing data unless otherwise noted.

Estimates of insulin secretion (Stumvoll first- and secondphase estimates [14]) and insulin sensitivity (Matsuda index [15]) were calculated using results from the HAPO OGTTs. Measures of insulin were not available; therefore, C-peptide (secreted 1:1 with insulin, but present in the serum at approximately sixfold higher concentrations because of lower hepatic extraction), assayed on fasting and $1 \mathrm{~h}$ OGTT samples, was substituted for insulin in these calculations [14, 16, 17]. The Stumvoll first- and second-phase insulin secretion estimates were highly correlated (Spearman's correlation $\rho=0.995$; $p<0.001$ ); in subsequent analyses, the Stumvoll first-phase estimate was used.

An abbreviated version of the Matsuda index of insulin sensitivity, as previously validated by Radaelli et al [17], was used. The oral disposition index (DI) was calculated as the product of the Stumvoll estimate of first-phase insulin secretion and insulin sensitivity as calculated using the Matsuda index, allowing correction of insulin secretion for prevailing insulin sensitivity. Estimates of insulin secretion, sensitivity and DI were subsequently transformed into $z$ scores to allow equal rating in regression estimates. We used the 
IADPSG criteria [4] to classify each woman as having or not having GDM [4]. To allow for comparison with the initial publication by Powe et al [6], we calculated the 25 th centile for insulin secretion (Stumvoll first phase) and insulin sensitivity (Matsuda index) for women without IADPSG-defined GDM. We then classified women with GDM as having an 'impairment' if a variable was less than the 25th centile for women without GDM. This produced four groups of women with GDM: GDM-secretion (impaired insulin secretion only), GDM-sensitivity (impaired insulin sensitivity only), GDMmixed (both impaired insulin secretion and impaired insulin sensitivity) and GDM-no defect (both insulin secretion and insulin sensitivity above the 25 th centile for women without GDM, but with GDM by the IADPSG criteria).

Baseline characteristics and outcomes are presented and compared between the GDM and non-GDM groups, using Mann-Whitney $U$ test or Student's $t$ test for continuous variables and Fisher's exact test for categorical variables. We then compared the association of: (1) GDM classification (GDM yes/no); (2) GDM subtype (Powe et al [6]); and (3) DI and Matsuda $z$ scores, with obstetric and neonatal outcomes using a logistic regression model. Receiver operating characteristic (ROC) curves using the predicted risk of adverse outcomes were then generated for each of the measures (1, 2 and 3) and the area under the ROC curve (AUROC) was calculated. The AUROCs were then compared [18]. Subsequently, we created a sequence of multivariable logistic regression models relating insulin sensitivity (Matsuda $z$ score) and DI $z$ score with outcomes in the presence of clinical and demographic variables. Model 1 included the Matsuda $z$ score and DI $z$ score; Model 2 added the mean OGTT $z$ score; and Model 3 added maternal BMI, ethnicity, age, height, smoking, parity, family history of diabetes, mean arterial BP and GWG group. HAPO study centre was not included because of collinearity with ethnicity. Adjusted ORs for the Matsuda $z$ score and DI $z$ score are reported along with $95 \%$ CIs and $p$ values. Two-tailed $p$ values of 0.05 were considered significant, with correction for multiple comparisons where required. Analyses were conducted using Stata/SE version 14 (StataCorp, USA).

\section{Results}

A total of 6337 women were included in the analysis: 1902 (30.0\%) from Bellflower, 1584 (25.0\%) from Hong Kong, $1425(22.5 \%)$ from Brisbane, 782 (12.3\%) from Cleveland and $644(10.2 \%)$ from Newcastle. The reported ethnicity distribution was $39.9 \%$ white, $26.2 \%$ Asian, $25.4 \%$ Hispanic, $4.9 \%$ black and $3.6 \%$ other. Of the 6337 women, $1090(17.2 \%)$ fulfilled the IADPSG criteria for GDM. Table 1 shows the maternal characteristics and obstetric and neonatal outcomes, both for the entire cohort and categorised (post hoc) by IADPSG GDM status. Figure 1 shows the relationship between insulin sensitivity as estimated by the Matsuda index and insulin secretion as estimated by Stumvoll first-phase estimate. As predicted from previous work [19], this relationship followed a hyperbolic pattern with the fitted exponential curve lying closer to the origin for women with GDM, confirming that for a given level of insulin sensitivity, women without GDM displayed higher insulin secretion.

Table 2 presents the predictive ability of: (1) dichotomous IADPSG GDM classification (yes/no); (2) Powe et al GDM subtypes; and (3) continuous analysis of insulin secretion and sensitivity using the DI and the Matsuda index to predict a range of pregnancy outcomes. All models poorly predicted neonatal hypoglycaemia. GDM subtype [6] offered superior prediction to IADPSG GDM only for neonatal hyperinsulinaemia and pregnancy-related hypertension. However, the DI and Matsuda score significantly increased the AUROC and improved prediction for most outcomes.

Figure 2 presents ROC curves for the logistic regression models as outlined in the Methods section, namely Model 1 (Matsuda and DI $z$ scores), Model 2 (Model 1 plus mean OGTT $z$ score) and Model 3 (Model 2 plus maternal clinical characteristics) for four important outcome variables: LGA, neonatal adiposity (per cent body fat $>90$ th centile), neonatal hyperinsulinaemia and pregnancy-related hypertension. The three models each produced a similar ROC curve for neonatal hyperinsulinaemia, but the addition of baseline maternal clinical characteristics in Model 3 greatly enhanced predictive power for LGA, neonatal adiposity and pregnancy-related hypertension.

Table 3 presents a stringent analysis of the relationship between insulin secretion or sensitivity and pregnancy outcomes, using the same models as outlined above. In the table, we have tabulated the relationships (aOR per SD increment) between the DI and Matsuda $z$ scores and the three outcome variables chosen by the IADPSG consensus panel [4] to develop GDM diagnostic criteria (namely LGA, neonatal adiposity and neonatal hyperinsulinaemia). The DI $z$ scores were generally significantly related to these outcomes on univariate analysis (Model 1), while the Matsuda $z$ scores were not. After Model 2 correction for mean OGTT $z$ score, the DI $z$ score remained significantly related to LGA, while the Matsuda $z$ score was significantly related to neonatal hyperinsulinaemia. After Model 3 adjustment, only the relationship between the Matsuda $z$ score and neonatal hyperinsulinaemia retained significance. Finally, electronic supplementary material (ESM) Table 1 shows baseline characteristics and outcomes using the subgroups proposed by Powe et al [6].

\section{Discussion}

In this large cohort study of 6337 women from the HAPO study, $17.2 \%$ fulfilled the IADPSG criteria for GDM, which was comparable with the total HAPO cohort [20]. We have 
Table 1 Maternal characteristics and obstetric and neonatal outcomes of 6337 women and their offspring by IADPSG GDM classification

\begin{tabular}{|c|c|c|c|c|}
\hline Variable & All participants & No GDM & GDM & $p$ value \\
\hline$n$ & 6337 & 5247 & 1090 & \\
\hline Maternal age, years & $29.4 \pm 5.4$ & $29.1 \pm 5.3$ & $30.9 \pm 5.5$ & $<0.001$ \\
\hline Maternal height, $\mathrm{cm}$ & $162 \pm 7$ & $162 \pm 7$ & $161 \pm 7$ & $<0.001$ \\
\hline Maternal BMI, $\mathrm{kg} / \mathrm{m}^{2}$ & $27.0(24.2-31.0)$ & $26.6(24.0-30.2)$ & $29.7(26.1-34.1)$ & $<0.001$ \\
\hline \multicolumn{5}{|l|}{ Maternal BMI by WHO category } \\
\hline Underweight & $789(12.5)$ & $725(13.8)$ & $64(5.9)$ & \multirow[t]{4}{*}{$<0.001$} \\
\hline Normal weight & $3060(48.3)$ & $2659(50.7)$ & $401(36.8)$ & \\
\hline Overweight & $1416(22.3)$ & $1121(21.4)$ & $295(27.1)$ & \\
\hline Obese & $1072(16.9)$ & $742(14.1)$ & $330(30.3)$ & \\
\hline GWG, kg & $14.1(10.2-18.0)$ & $14.0(10.2-18.0)$ & $14.1(10.0-18.2)$ & 0.770 \\
\hline \multicolumn{5}{|l|}{ GWG by IOM recommendation $^{\mathrm{a}}$} \\
\hline$<\mathrm{IOM}$ recommendation & $1340(22.2)$ & $1173(23.4)$ & $167(16.2)$ & \multirow[t]{3}{*}{$<0.001$} \\
\hline$=\mathrm{IOM}$ recommendation & $1864(30.9)$ & $1568(31.3)$ & $296(28.8)$ & \\
\hline$>\mathrm{IOM}$ recommendation & $2829(46.9)$ & $2264(45.2)$ & $565(55.0)$ & \\
\hline Smoking & $465(7.3)$ & $381(7.3)$ & $84(7.7)$ & 0.61 \\
\hline Alcohol use & $503(7.9)$ & $444(8.5)$ & $59(5.4)$ & $<0.001$ \\
\hline Multiparous & $3362(53.1)$ & $2700(51.5)$ & $662(60.7)$ & $<0.001$ \\
\hline \multicolumn{5}{|l|}{ Ethnicity } \\
\hline White & $2529(39.9)$ & $2156(41.1)$ & $373(34.2)$ & \multirow[t]{5}{*}{$<0.001$} \\
\hline Black & $313(4.9)$ & $252(4.8)$ & $61(5.6)$ & \\
\hline Hispanic & $1610(25.4)$ & $1232(23.5)$ & $378(34.7)$ & \\
\hline Asian & $1660(26.2)$ & $1416(27.0)$ & $244(22.4)$ & \\
\hline Other & $225(3.6)$ & $191(3.6)$ & $34(3.1)$ & \\
\hline Family history of diabetes & $1549(24.4)$ & $1191(22.7)$ & $358(32.8)$ & $<0.001$ \\
\hline Family history of hypertension & $2528(39.9)$ & $2066(39.4)$ & $462(42.4)$ & 0.066 \\
\hline Mean arterial $\mathrm{BP}, \mathrm{mmHg}$ & $81.6 \pm 8.5$ & $81.1 \pm 8.4$ & $84.4 \pm 8.6$ & $<0.001$ \\
\hline Fasting glucose, mmol/l & $4.5 \pm 0.4$ & $4.4 \pm 0.3$ & $5.0 \pm 0.4$ & $<0.001$ \\
\hline $1 \mathrm{~h}$ glucose, $\mathrm{mmol} / \mathrm{l}$ & $7.5 \pm 1.7$ & $7.1 \pm 1.4$ & $9.5 \pm 1.6$ & $<0.001$ \\
\hline $2 \mathrm{~h}$ glucose, $\mathrm{mmol} / \mathrm{l}$ & $6.3 \pm 1.3$ & $6.0 \pm 1.0$ & $7.7 \pm 1.5$ & $<0.001$ \\
\hline Mean OGTT $z$ score & $0.05 \pm 0.77$ & $-0.19 \pm 0.57$ & $1.17 \pm 0.60$ & $<0.001$ \\
\hline $\mathrm{HbA}_{1 \mathrm{c}}, \mathrm{mmol} / \mathrm{mol}$ & $29.3 \pm 4.1$ & $28.8 \pm 4.0$ & $31.4 \pm 4.2$ & $<0.001$ \\
\hline $\mathrm{HbA}_{1 \mathrm{c}}, \%$ & $4.8 \pm 0.4$ & $4.8 \pm 0.4$ & $5.0 \pm 0.4$ & $<0.001$ \\
\hline \multicolumn{5}{|l|}{ Outcomes } \\
\hline $\begin{array}{l}\text { Pregnancy-related } \\
\text { hypertension }(n=6143)\end{array}$ & $1000(16.3)$ & $747(14.6)$ & $253(24.7)$ & $<0.001$ \\
\hline Preterm delivery & $369(5.8)$ & $278(5.3)$ & $91(8.3)$ & $<0.001$ \\
\hline Birthweight, $\mathrm{g}(n=6319)$ & $3382 \pm 517$ & $3360 \pm 508$ & $3490 \pm 544$ & $<0.001$ \\
\hline LGA $(n=6319)$ & $601(9.5)$ & $427(8.2)$ & $174(16.0)$ & $<0.001$ \\
\hline Primary Caesarean section $(n=5584)$ & $1009(18.1)$ & $795(17.0)$ & $214(23.4)$ & $<0.001$ \\
\hline Neonatal hyperinsulinaemia $(n=5570)$ & $486(8.7)$ & $328(7.1)$ & $158(16.3)$ & $<0.001$ \\
\hline Neonatal hypoglycaemia $(n=4750)$ & $832(17.5)$ & $668(17.1)$ & $164(19.5)$ & 0.099 \\
\hline Neonatal adiposity $(n=5108)$ & $491(9.6)$ & $354(8.3)$ & $137(16.1)$ & $<0.001$ \\
\hline Sum of skinfolds $(n=5136)$ & $12.6 \pm 2.8$ & $12.4 \pm 2.7$ & $13.8 \pm 3.1$ & $<0.001$ \\
\hline Skin fold LGA $(n=5136)$ & $480(9.3)$ & $330(7.7)$ & $150(17.4)$ & $<0.001$ \\
\hline
\end{tabular}

Data are means $\pm \mathrm{SD}$, medians (IQR) or $n(\%)$

$p$ values refer to the comparison of women with vs without GDM (IADPSG criteria)

${ }^{a}$ Four values missing

IOM, Institute of Medicine 


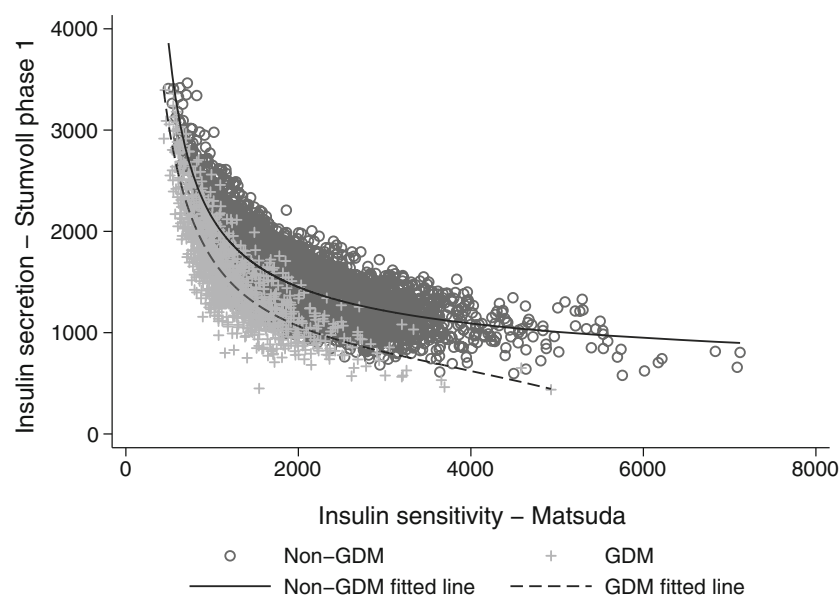

Fig. 1 Relationship between insulin sensitivity (measured by the modified Matsuda index) and insulin secretion (measured by the Stumvoll first-phase estimate)

demonstrated that our modified Matsuda (insulin sensitivity) and Stumvoll first phase (insulin secretion) measures conformed to the expected hyperbolic relationship and showed appropriate patterns when comparing women with vs without GDM. The hyperbolic relationship between these two measurements underlines the problem of dividing women with GDM into subgroups based solely on impairment of insulin sensitivity or insulin secretion. Clearly, even a woman with very markedly impaired insulin sensitivity may remain normoglycaemic if her insulin secretion is 'perfect', and varying degrees of both defects are likely to be present in many individuals. Our data also demonstrate that using these measures as continuous variables improves the prediction of a range of pregnancy outcomes, as compared with a dichotomous GDM classification or the empirical four-category analysis of Powe et al [6].

However, although statistically significant, the magnitude of improved outcome prediction was only modest (highest final AUROC 0.688). The additional ROC curves presented in Fig. 2 demonstrate the relevance of adding additional clinical variables, including maternal BMI, to potential predictive models. These clinical variables greatly enhanced the predictive power of the statistical model and offer the advantage of being readily ascertained in routine care. Thus, we suggest that measures of insulin sensitivity or insulin secretion, which require additional laboratory measures (insulin or C-peptide), cannot stand alone. Although they offer some insights into the pathophysiology of GDM, our data suggest that they should be evaluated together with additional clinical variables, rather than in isolation, to determine their overall utility and both clinical and health economic 'value' in enhancing the prediction of outcomes. Although beyond the scope of our current paper, it appears unlikely that their routine clinical use would greatly enhance outcome prediction. Furthermore, although one could reasonably hypothesise that stratifying women with GDM according to their primary impairment could lead to more targeted medical treatment during pregnancy (e.g. metformin as initial therapy for those with primary defects in insulin sensitivity vs insulin as initial therapy for those with primary defects in insulin secretion), there is currently no evidence that such an approach would improve clinical outcomes.

Interestingly, the analyses in Table 3 demonstrate that the DI, but not the Matsuda index, relates closely to LGA and excess fetal adiposity, two outcomes chosen by the IADPSG to define GDM. This suggests that it is not reduced insulin sensitivity per se, but rather a failure to adapt to this by appropriate augmentation of insulin secretion, that most closely relates to fetal growth. As predicted, this relationship was attenuated after correction for OGTT measures (Model 2), which relate to the predicted downstream consequence of a low DI, namely (generally mild) hyperglycaemia. By contrast, the Matsuda index related more closely to neonatal

Table 2 Comparison of the predictive ability of: (1) IADPSG GDM classification; (2) GDM subtype; and (3) DI combined with the Matsuda index, using AUROC

\begin{tabular}{|c|c|c|c|c|c|c|}
\hline \multirow[t]{2}{*}{ Outcome } & \multicolumn{2}{|c|}{ IADPSG GDM } & \multicolumn{2}{|c|}{ GDM subtype } & \multicolumn{2}{|c|}{ DI + Matsuda score } \\
\hline & AUROC & $95 \% \mathrm{CI}$ & AUROC & $95 \% \mathrm{CI}$ & AUROC & $95 \% \mathrm{CI}$ \\
\hline $\operatorname{LGA}^{\mathrm{b}, \mathrm{c}}$ & 0.565 & $0.546,0.584$ & 0.567 & $0.548,0.586$ & 0.632 & $0.609,0.655$ \\
\hline Neonatal adiposity ${ }^{\mathrm{b}, \mathrm{c}}$ & 0.562 & $0.542,0.583$ & 0.564 & $0.543,0.584$ & 0.630 & $0.604,0.656$ \\
\hline Neonatal hyperinsulinaemia $\mathrm{a}^{\mathrm{a}-\mathrm{c}}$ & 0.583 & $0.561,0.604$ & 0.586 & $0.564,0.608$ & 0.688 & $0.662,0.712$ \\
\hline Pregnancy-related hypertension ${ }^{\mathrm{a}-\mathrm{c}}$ & 0.552 & $0.537,0.566$ & 0.567 & $0.553,0.581$ & 0.669 & $0.651,0.687$ \\
\hline Neonatal hypoglycaemia & 0.512 & $0.497,0.527$ & 0.517 & $0.502,0.532$ & 0.521 & $0.499,0.543$ \\
\hline Primary Caesarean section ${ }^{\mathrm{b}, \mathrm{c}}$ & 0.529 & $0.516,0.543$ & 0.536 & $0.522,0.550$ & 0.577 & $0.557,0.596$ \\
\hline Preterm $^{\mathrm{b}}$ & 0.540 & $0.517,0.562$ & 0.543 & $0.520,0.566$ & 0.572 & $0.541,0.603$ \\
\hline
\end{tabular}

${ }^{\mathrm{a}} p<0.05$ for IADPSG GDM vs GDM subtype

${ }^{\mathrm{b}} p<0.05$ for IADPSG GDM vs DI + Matsuda score

${ }^{\mathrm{c}} p<0.05$ for GDM subtype vs DI + Matsuda score 
Fig. 2 ROC curves comparing Model 1 (Matsuda $z$ score and DI $z$ score), Model 2 (Model 1 plus mean OGTT $z$ score) and Model 3 (Model 2 plus maternal BMI, ethnicity, age, height, smoking, parity, family history of diabetes, mean arterial BP and GWG group). (a) LGA; (b) neonatal per cent body fat $>90$ th centile; (c) neonatal hyperinsulinaemia; and (d) pregnancy-related hypertension a

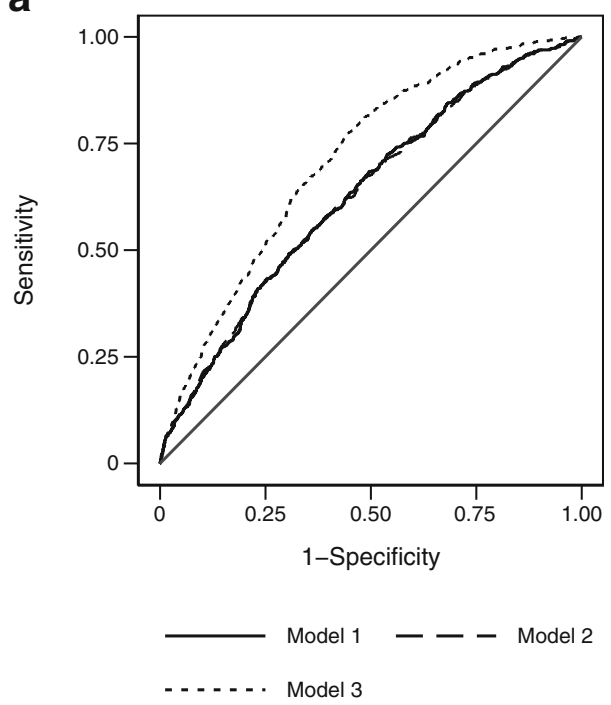

C

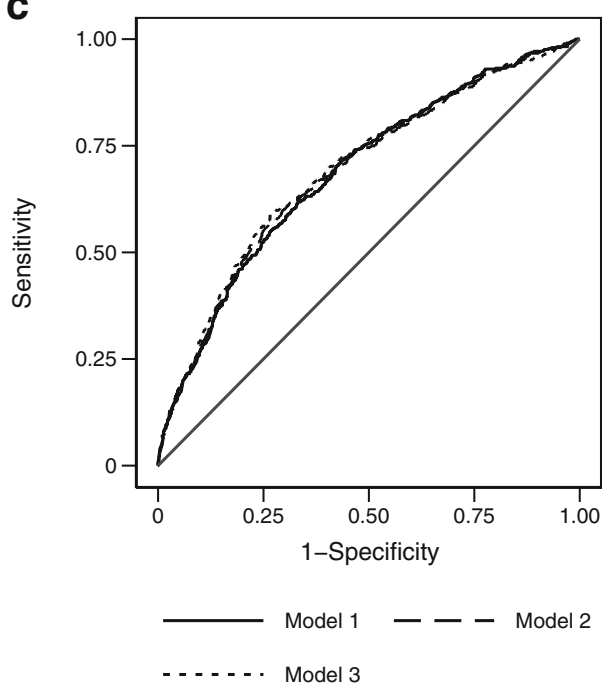

b

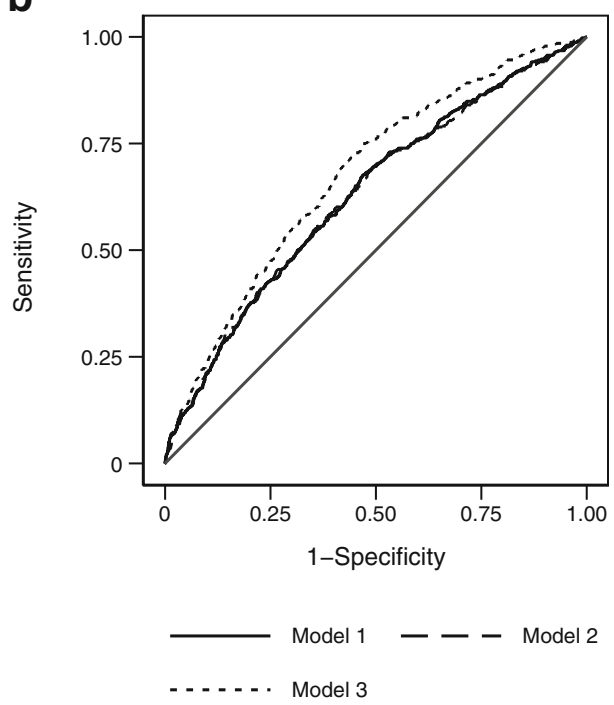

d

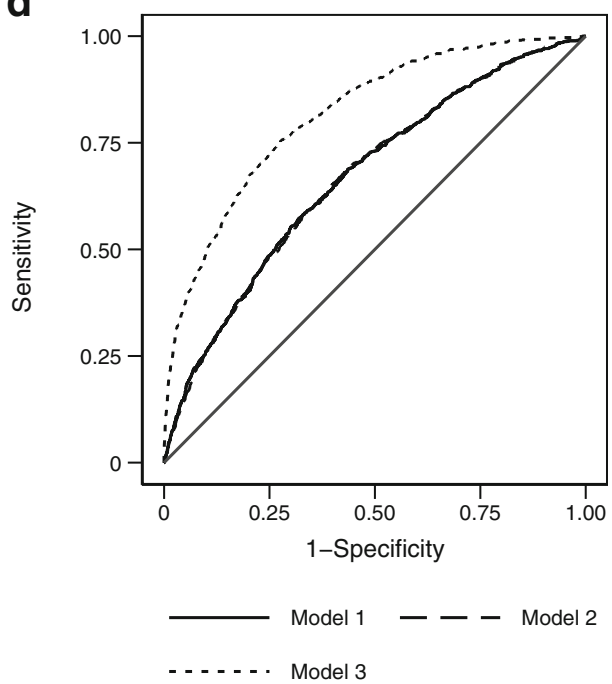

Table 3 Relationships (aOR per SD increment) between the DI and Matsuda $z$ scores and LGA, neonatal adiposity and neonatal hyperinsulinaemia

\begin{tabular}{|c|c|c|c|c|c|c|c|c|c|c|}
\hline \multirow[t]{2}{*}{ Outcome } & \multirow[t]{2}{*}{ Model } & \multicolumn{3}{|c|}{ Model 1} & \multicolumn{3}{|c|}{ Model 2} & \multicolumn{3}{|c|}{ Model 3} \\
\hline & & $\mathrm{aOR}$ & $95 \% \mathrm{CI}$ & $P$ & $\mathrm{aOR}$ & $95 \% \mathrm{CI}$ & $p$ & $\mathrm{aOR}$ & $95 \% \mathrm{CI}$ & $p$ \\
\hline \multirow[t]{2}{*}{ LGA } & DI & 0.59 & $0.51,0.69$ & $<0.001$ & 0.71 & $0.52,0.98$ & 0.035 & 0.75 & $0.54,1.06$ & 0.098 \\
\hline & Matsuda & 0.97 & $0.83,1.13$ & 0.705 & 0.92 & $0.78,1.10$ & 0.366 & 0.96 & $0.79,1.16$ & 0.656 \\
\hline \multirow[t]{2}{*}{ Neonatal adiposity } & DI & 0.62 & $0.52,0.74$ & $<0.001$ & 0.87 & $0.61,1.22$ & 0.412 & 0.88 & $0.61,1.25$ & 0.467 \\
\hline & Matsuda & 0.96 & $0.80,1.14$ & 0.613 & 0.87 & $0.72,1.06$ & 0.161 & 0.95 & $0.77,1.18$ & 0.663 \\
\hline \multirow[t]{2}{*}{ Neonatal hyperinsulinaemia } & DI & 0.55 & $0.46,0.66$ & $<0.001$ & 0.90 & $0.62,1.30$ & 0.569 & 0.93 & $0.63,1.36$ & 0.694 \\
\hline & Matsuda & 0.78 & $0.65,0.94$ & 0.010 & 0.68 & $0.55,0.85$ & $<0.001$ & 0.69 & $0.55,0.87$ & 0.002 \\
\hline
\end{tabular}

aOR, change per SD change in the specified independent variable

Model 1: DI $z$ score and Matsuda $z$ score

Model 2: Model 1 plus mean OGTT $z$ score

Model 3: Model 2 plus maternal BMI (categorical), ethnicity, age, height, smoking, parity, family history of diabetes, mean arterial BP and GWG (categorical) 
hyperinsulinaemia and this relationship was strengthened after Model 2 and 3 corrections. The significance of this observation is less clear, but it might represent residual confounding.

Our categorical analysis, following the methods of Powe et al [6] (ESM Table 1), revealed that almost two-thirds of women with GDM had impaired insulin sensitivity, a quarter had impaired insulin secretion and approximately $10 \%$ displayed both impairments. Women with GDM and impaired insulin sensitivity differed at baseline from women without GDM. They were older, had a higher BMI and were more likely to be multiparous and have a first-degree family history of diabetes, all characteristics previously reported in women with GDM caused by impaired insulin sensitivity [7, 21, 22]. The GDM-sensitivity group had higher GWG and clearly demonstrated higher rates of adverse obstetric and neonatal outcomes compared with women without GDM, including primary Caesarean delivery, LGA offspring, pregnancyrelated hypertension, neonatal hyperinsulinaemia and neonatal adiposity. In contrast to findings from previous studies, women in the GDM-secretion group also showed higher risks of LGA offspring, neonatal hyperinsulinaemia and neonatal adiposity compared with women without GDM. The $9.9 \%$ of women with GDM with both impairments displayed risks similar to those of the women in GDM-sensitivity group. This contrasts with the findings from a study by Liu et al from China $(n=206)$, which characterised women with GDM according to impairments evaluated by the Matsuda index and the DI [23]. Liu et al reported that their GDM-mixed group had a higher risk of LGA offspring and of a composite adverse outcome comprising LGA, neonatal hypoglycaemia and Caesarean delivery, similar to that used by Powe et al [6]. However, these risks were no longer significant after adjustment for age and BMI [23]. Compared with the studies by Powe et al ( $n=67$ women with GDM) [6] and Benhalima et al ( $n=231$ women with GDM) [7], which reported IADPSG-defined GDM prevalences of $8.3 \%$ and $12.4 \%$, respectively, we found that more than $17 \%$ of women had GDM, comparable with the overall HAPO cohort [9]. This difference could be explained by differences in ethnic composition and mean age of the cohorts.

Our study has several strengths. We considered variations in insulin sensitivity and secretion and a broad range of obstetric and neonatal outcomes in a blinded and untreated cohort. Our cohort was much larger than those of previous reports, allowing us to adjust for multiple confounders and compare obstetric and neonatal outcomes. In addition, the broad ethnic diversity of our cohort increases the generalisability of our findings. Furthermore, we have detailed data on neonatal anthropometry, enabling us to evaluate both overall size at birth and adiposity. A major strength of our study is a lack of confounding by GDM treatment. In contrast, up to $42 \%$ of women in previous studies received insulin treatment, which would be predicted to attenuate any potential influence of impaired insulin secretion on neonatal outcomes, including neonatal hypoglycaemia [24].

Our study was limited by the fact that the HAPO study did not assay plasma insulin; therefore, we used plasma C-peptide (with appropriate corrections) in our calculations of the Stumvoll and Matsuda variables. The use of the Matsuda index in pregnancy with substitution of C-peptide for insulin has been validated, as has the use of the truncated version (fasting and $1 \mathrm{~h} \mathrm{C}$-peptide only) used in our study [17]. Recent reports presented as abstracts support the validity of the Stumvoll index of insulin secretion in pregnancy, measured against the IVGTT [25, 26], but use of C-peptide instead of insulin in the Stumvoll index, although logical, has not been formally validated. A further limitation of our study relates to the HAPO study policy, which, for reasons of participant safety, unblinded and excluded from further analysis those women with more marked hyperglycaemia (fasting blood glucose $>5.8 \mathrm{mmol} / \mathrm{l}$ and/or $2 \mathrm{~h}$ glucose $>11.1 \mathrm{mmol} / 1$ at OGTT or any glucose $<2.5 \mathrm{mmol} / \mathrm{l}$ ). These excluded women (who comprised only $2.9 \%$ of the entire HAPO cohort) [9] would be predicted to be more likely to experience adverse pregnancy outcomes; therefore, we might have underestimated overall outcome frequencies in our cohort.

In conclusion, our study clearly outlines the relationship between continuous measurements of insulin secretion and insulin sensitivity with pregnancy outcomes in a multi-ethnic population. Although the use of such measures does provide a modest improvement in outcome prediction, the use of basic clinical variables appears to have stronger effects. This favours considering the 'risk engine' approach to improve prediction of pregnancy outcomes [27]. In future studies, it would also be valuable to prospectively determine whether stratification of GDM according to underlying pathophysiology might assist in pregnancy-related treatment decisions, and whether GDM subtypes have any relationship to long-term metabolic consequences (especially the risks of adiposity and hyperglycaemia) for both mothers and their offspring.

Acknowledgements Abstracts related to this dataset have been presented previously at the EASD annual meeting, Berlin, Germany, September 2018; the ADIPS 2018 Annual Scientific Meeting, Adelaide, South Australia, Australia, August 2018; the ADA annual meeting, San Francisco, CA, USA, June 2019; and the DPSG annual meeting, Graz, Austria, September 2019.

Data availability The datasets generated during and/or analysed during the current study are available from the corresponding author on reasonable request, subject to approval from the HAPO Study Steering Committee.

Funding LRM and HDM received funding from the Danish Diabetes Academy, founded by the Novo Nordisk Foundation. The funder was not involved in the design of the study; the collection, analysis or interpretation of data; or writing the report; and did not impose any restrictions regarding the publication of the report. 
Authors' relationships and activities The authors declare that there are no relationships or activities that might bias, or be perceived to bias, their work.

Contribution statement LRM planned the analysis, contributed to data interpretation and wrote the manuscript. KSG planned the analysis and conducted the statistical analysis. HDM planned the analysis, contributed to data interpretation and supervised the manuscript writing. RCWM, WHT, PMC, DAS, JL and HDM all contributed substantially to data acquisition. All authors contributed to the discussion and revised the manuscript critically for important intellectual content. All authors approved the final version of the manuscript. HDM is the guarantor of this work, has access to all data, and takes responsibility for the integrity of data and accuracy of data analysis.

\section{References}

1. American Diabetes Association (2020) 14. Management of diabetes in pregnancy: standards of medical care in diabetes 2020. Diabetes Care 43(Suppl 1):S183-s192. https://doi.org/10.2337/dc20-S014

2. Kampmann U, Madsen LR, Skajaa GO, Iversen DS, Moeller N, Ovesen P (2015) Gestational diabetes: a clinical update. World J Diabetes 6(8):1065-1072. https://doi.org/10.4239/wjd.v6.i8.1065

3. McIntyre HD, Catalano P, Zhang C, Desoye G, Mathiesen ER, Damm P (2019) Gestational diabetes mellitus. Nat Rev Dis Primers 5(1):47. https://doi.org/10.1038/s41572-019-0098-8

4. Metzger BE, Gabbe SG, Persson B et al (2010) International association of diabetes and pregnancy study groups recommendations on the diagnosis and classification of hyperglycemia in pregnancy. Diabetes Care 33(3):676-682. https://doi.org/10.2337/dc09-1848

5. Sheiner E, Kapur A, Retnakaran R et al (2019) FIGO (International Federation of Gynecology and Obstetrics) Postpregnancy Initiative: long-term maternal implications of pregnancy complicationsfollow-up considerations. Int J Gynaecol Obstet 147(Suppl 1):131. https://doi.org/10.1002/ijgo.12926

6. Powe CE, Allard C, Battista MC et al (2016) Heterogeneous contribution of insulin sensitivity and secretion defects to gestational diabetes mellitus. Diabetes Care 39(6):1052-1055. https://doi.org/ $10.2337 / \mathrm{dc} 15-2672$

7. Benhalima K, Van Crombrugge P, Moyson C et al (2019) Characteristics and pregnancy outcomes across gestational diabetes mellitus subtypes based on insulin resistance. Diabetologia 62(11): 2118-2128. https://doi.org/10.1007/s00125-019-4961-7

8. Sokup A, Ruszkowska-Ciastek B, Goralczyk K, Walentowicz M, Szymanski M, Rosc D (2013) Insulin resistance as estimated by the homeostatic method at diagnosis of gestational diabetes: estimation of disease severity and therapeutic needs in a population-based study. BMC Endocr Disord 13:21. https://doi.org/10.1186/1472-6823-13-21

9. Metzger BE, Lowe LP, Dyer AR et al (2008) Hyperglycemia and adverse pregnancy outcomes. N Engl J Med 358(19):1991-2002. https://doi.org/10.1056/NEJMoa0707943

10. HAPO Study Cooperative Research Group (2002) The Hyperglycemia and Adverse Pregnancy Outcome (HAPO) Study. Int J Gynaecol Obstet 78(1):69-77. https://doi.org/10.1016/s0020-7292(02)00092-9

11. HAPO Study Cooperative Research Group (2010) Hyperglycaemia and Adverse Pregnancy Outcome (HAPO) Study: associations with maternal body mass index. BJOG 117(5):575-584. https://doi.org/ 10.1111/j.1471-0528.2009.02486.x

12. Catalano PM, McIntyre HD, Cruickshank JK et al (2012) The hyperglycemia and adverse pregnancy outcome study: associations of GDM and obesity with pregnancy outcomes. Diabetes Care 35(4):780-786. https://doi.org/10.2337/dc11-1790

13. Badon SE, Dyer AR, Josefson JL (2014) Gestational weight gain and neonatal adiposity in the Hyperglycemia and Adverse Pregnancy Outcome study-North American region. Obesity (Silver Spring) 22(7):1731-1738. https://doi.org/10.1002/oby.20742

14. Stumvoll M, Van Haeften T, Fritsche A, Gerich J (2001) Oral glucose tolerance test indexes for insulin sensitivity and secretion based on various availabilities of sampling times. Diabetes Care 24(4):796-797. https://doi.org/10.2337/diacare.24.4.796

15. Matsuda M, DeFronzo RA (1999) Insulin sensitivity indices obtained from oral glucose tolerance testing: comparison with the euglycemic insulin clamp. Diabetes Care 22(9):1462-1470. https://doi.org/10.2337/diacare.22.9.1462

16. Larsen PB, Linneberg A, Hansen T, Friis-Hansen L (2017) Reference intervals for C-peptide and insulin derived from a general adult Danish population. Clin Biochem 50(7-8):408-413. https://doi.org/10.1016/j.clinbiochem.2016.12.004

17. Radaelli T, Farrell KA, Huston-Presley L et al (2010) Estimates of insulin sensitivity using glucose and C-peptide from the hyperglycemia and adverse pregnancy outcome glucose tolerance test. Diabetes Care 33(3):490-494. https://doi.org/10.2337/dc09-1463

18. DeLong ER, DeLong DM, Clarke-Pearson DL (1988) Comparing the areas under two or more correlated receiver operating characteristic curves: a nonparametric approach. Biometrics 44(3):837845. https://doi.org/10.2307/2531595

19. Buchanan TA (2001) Pancreatic B-cell defects in gestational diabetes: implications for the pathogenesis and prevention of type 2 diabetes. J Clin Endocrinol Metab 86(3):989-993. https://doi.org/ 10.1210/jcem.86.3.7339

20. Sacks DA, Hadden DR, Maresh M et al (2012) Frequency of gestational diabetes mellitus at collaborating centers based on IADPSG consensus panel-recommended criteria: the Hyperglycemia and Adverse Pregnancy Outcome (HAPO) Study. Diabetes Care 35(3):526-528. https://doi.org/10.2337/dc11-1641

21. Yamada H, Hirayama Kato E, Tsuruga R et al (2001) Insulin response patterns contribute to different perinatal risks in gestational diabetes. Gynecol Obstet Investig 51(2):103-109. https://doi.org/ $10.1159 / 000052903$

22. Sun YY, Juan J, Xu QQ, Su RN, Hirst JE, Yang HX (2020) Increasing insulin resistance predicts adverse pregnancy outcomes in women with gestational diabetes mellitus. J Diabetes 12(6):438 446. https://doi.org/10.1111/1753-0407.13013

23. Liu Y, Hou W, Meng X et al (2018) Heterogeneity of insulin resistance and beta cell dysfunction in gestational diabetes mellitus: a prospective cohort study of perinatal outcomes. J Transl Med 16(1): 289

24. Saisho Y, Miyakoshi K, Tanaka M et al (2010) Beta cell dysfunction and its clinical significance in gestational diabetes. Endocr $\mathrm{J}$ 57(11):973-980. https://doi.org/10.1507/endocrj.k10e-231

25. Powe CE, Locascio JJ, Catalano P (2020) Changes in OGTT-based measures of insulin secretory response across pregnancy. Diabetes 69(Suppl 1). https://doi.org/10.2337/db20-1384-P

26. Powe CE, Gordesky LH, Catalano PM (2020) OR08-02 Do OGTTbased insulin secretory response measures approximate 1 (st) phase insulin response in pregnant women? J Endocr Soc 4(Suppl 1): OR08-02. https://doi.org/10.1210/jendso/bvaa046.245

27. McIntyre HD, Gibbons KS, Lowe J, Oats JJN Development of a risk engine relating maternal glycemia and body mass index to pregnancy outcomes. Diabetes Res Clin Pract 139:331-338. https://doi.org/10.1016/j.diabres.2018.02.036

Publisher's note Springer Nature remains neutral with regard to jurisdictional claims in published maps and institutional affiliations. 